\title{
Obstructive sleep apnoea as a risk factor for incident metabolic syndrome: a joined Episono and HypnoLaus prospective cohorts study
}

\author{
Camila Hirotsu ${ }^{1,2,6}$, Jose Haba-Rubio ${ }^{2,6}$, Sonia M. Togeiro', \\ Pedro Marques-Vidal ${ }^{3}$, Luciano F. Drager ${ }^{4}$, Peter Vollenweider ${ }^{3}$, \\ Gérard Waeber ${ }^{3}$, Lia Bittencourt ${ }^{1}$, Sergio Tufik $^{1,7}$ and Raphael Heinzer ${ }^{2,5,7}$
}

Affiliations: ${ }^{1}$ Dept of Psychobiology, Universidade Federal de São Paulo (UNIFESP), São Paulo, Brazil. ${ }^{2}$ Center for Investigation and Research in Sleep (CIRS), University Hospital of Lausanne (CHUV), Lausanne, Switzerland. ${ }^{3}$ Dept of Internal Medicine, University Hospital of Lausanne (CHUV), Lausanne, Switzerland. ${ }^{4}$ Hypertension Unit, Heart Institute (InCor) and Renal Division, University of São Paulo Medical School, São Paulo, Brazil. ${ }^{5}$ Pulmonary Dept, University Hospital of Lausanne (CHUV), Lausanne, Switzerland. ${ }^{6}$ These two authors are joint first authors. ${ }^{7}$ These two authors are joint last authors.

Correspondence: Raphael Heinzer, Center for Investigation and Research in Sleep, University Hospital of Lausanne (CHUV), Lausanne University, Rue du Bugnon 46, 1011 Lausanne, Switzerland.

E-mail: raphael.heinzerdachuv.ch

@ERSpublications

OSA is an independent risk factor for incident metabolic syndrome (MetS) through mediation of nocturnal hypoxaemia in the general population. Conversely, MetS does not appear to be independently associated with incident OSA. http://ow.ly/ANKy30m2Vn4

Cite this article as: Hirotsu C, Haba-Rubio J, Togeiro SM, et al. Obstructive sleep apnoea as a risk factor for incident metabolic syndrome: a joined Episono and HypnoLaus prospective cohorts study. Eur Respir J 2018; 52: 1801150 [https://doi.org/10.1183/13993003.01150-2018].

ABSTRACT Cross-sectional studies have demonstrated that obstructive sleep apnoea (OSA) and metabolic syndrome (MetS) are often associated, but whether a temporal relationship exists is unknown. We aimed to investigate the effect of OSA on the risk of developing MetS in the general population.

A prospective study was conducted combining two population-based samples: Episono (Brazil) and HypnoLaus (Switzerland). MetS was assessed according to unified criteria. Polysomnography (PSG) was performed at baseline and follow-up in Episono, and at baseline in HypnoLaus. OSA was defined according to the apnoea-hypopnoea index as mild $\left(\geqslant 5-<15\right.$ events $\left.h^{-1}\right)$ and moderate-to-severe $\left(\geqslant 15\right.$ events $\left.\cdot \mathrm{h}^{-1}\right)$. We included 1853 participants (mean \pm SD age $52 \pm 13$ years, $56 \%$ female) without MetS at baseline.

After mean \pm SD $6 \pm 1$ years, 318 (17.2\%) participants developed MetS. Moderate-to-severe OSA was independently associated with incident MetS (OR 2.58, 95\% CI 1.61-4.11) and increased the number of MetS components from baseline to follow-up through mediation of the percentage of time with arterial oxygen saturation $<90 \%$. Subset analysis in Episono confirmed that the increase in this parameter between baseline and follow-up PSGs represented a risk factor for incident MetS (OR 1.42, 95\% CI 1.04-1.95, for each $10 \%$ increase).

OSA is independently associated with an increased risk of developing MetS through mediation of nocturnal hypoxaemia in the general population. 


\section{Introduction}

Metabolic syndrome (MetS) is a worldwide major public health concern [1]. It is characterised by a cluster of interrelated cardiometabolic risk factors, such as abdominal obesity, atherogenic dyslipidaemia, high blood pressure and hyperglycaemia [2].

Previous investigations have demonstrated that obstructive sleep apnoea (OSA) and MetS tend to cluster in clinical populations [3, 4]. Considering that OSA independently predicts weight gain [5], it has been postulated that obesity could be the main mediator of the association between OSA and MetS [6, 7]. However, a meta-analysis of 10 cross-sectional studies revealed that OSA was significantly associated with MetS independently of body mass index (BMI) [8]. Intermittent hypoxaemia and sleep fragmentation due to multiple arousals are among the most plausible pathophysiological hypotheses linking OSA to MetS, as they could mediate oxidative stress, impair glucose and lipid metabolism, and contribute to a pro-inflammatory state [9].

The relationship between OSA and MetS could be bidirectional, with OSA increasing the risk of developing MetS or vice versa. It is therefore important to determine the temporal relationship between these two conditions.

Thus, the primary aim of the current study was to evaluate the impact of OSA on the incidence of MetS in a multiethnic sample combining two population-based samples (Episono in Brazil and HypnoLaus in Switzerland). Secondary aims included the analysis of the MetS components affected by OSA and the mediators of this association, as well as the possible bidirectional relationship between MetS and OSA (in the Episono cohort).

\section{Material and methods}

\section{Study design and population}

The sample was derived from two prospective population-based studies: Episono [10] and HypnoLaus [11] (a nested study of CoLaus/PsyCoLaus [12]). Episono and HypnoLaus sleep cohorts were designed to assess the prevalence and determinants of OSA in a general population from the cities of São Paulo, Brazil (August 2007 to January 2008) and Lausanne, Switzerland (September 2009 to June 2013), respectively. The design, sampling and procedures of both cohorts were described elsewhere [10, 11]. Briefly, 1074 individuals from Episono (1042 representative participants plus 32 substitutes) and 2162 individuals from HypnoLaus had a baseline examination including demographic, medical history, anthropometric and blood pressure measurements, blood sample collection for biological assays, subjective sleep assessment, and overnight full polysomnography (PSG).

After a mean follow-up of 7.9 years (Episono) and 5.2 years (HypnoLaus), participants underwent a second examination (July 2015 to April 2016 for Episono, n=712; May 2014 to May 2017 for HypnoLaus, $\mathrm{n}=1990$ ), in which demographic, clinical and biochemical parameters were reassessed.

\section{Ethics}

Episono was approved by the Ethics Committee of Universidade Federal de São Paulo (593/06 and 610514/14) and HypnoLaus by the Ethics Committee of the University of Lausanne (16/03 and 33/09). Written informed consent forms were completed and signed by all participants.

\section{Questionnaires}

Information about demographic data, socioeconomic and marital status, self-reported ethnicity, menopausal status, frequency of smoking and alcohol consumption, physical activity, current medications, and subjective aspects of sleep was collected using questionnaires in both cohorts. Self-reported sleep duration was obtained from the fourth question of the Pittsburgh Sleep Quality Index [13], while subjective sleepiness was assessed through the Epworth Sleepiness Scale (ESS) [14].

\section{Clinical data}

All measurements were made by trained observers using standard techniques. Body weight was measured in kilograms using a calibrated scale to within $0.1 \mathrm{~kg}$ (Seca, Hamburg, Germany (HypnoLaus/CoLaus) and InBody 720; Biospace, Cerritos, CA, USA (Episono)). Height was measured using a vertical stadiometer (Seca (HypnoLaus) and Professional Stadiometer; Sanny, São Paulo, Brazil (Episono)) to within $0.5 \mathrm{~cm}$. Waist (at the level of the umbilicus) circumference was measured to within $1 \mathrm{~mm}$ with plastic tape.

Systolic blood pressure (SBP) and diastolic blood pressure (DBP) were assessed in triplicate on the left arm at 5-min intervals with the participant seated and resting for at least $10 \mathrm{~min}$ using calibrated automated oscillometric sphygmomanometers (Omron HEM-907; Omron, Matsusaka, Japan (HypnoLaus) and Geratherm Desktop 995; Geratherm, Geschwenda, Germany (Episono)). The mean of the second and 
third measurements was used for analysis. Exceptionally in Episono, only one measurement of blood pressure was performed at baseline.

\section{Blood assays}

Overnight fasting blood samples were taken from the antecubital vein of each participant at the morning visit to the outpatient clinic (HypnoLaus) or after PSG (Episono). Glucose, total cholesterol, high-density lipoprotein (HDL) cholesterol and triglycerides were quantified by colorimetric assays as described previously $[10,12]$. Low-density lipoprotein (LDL) cholesterol was calculated through the Friedewald formula only if triglycerides were $<4.6 \mathrm{mmol} \cdot \mathrm{L}^{-1}$. C-reactive protein was measured by immunoassay in both cohorts. The assays were performed on fresh blood samples by the University Hospital of Lausanne Clinical Laboratory (Lausanne, Switzerland) and the Associação Fundo de Incentivo à Pesquisa (AFIP) Medicina Diagnóstica (São Paulo, Brazil).

\section{Polysomnography}

Participants of the Episono cohort were subjected to full-night PSG at both baseline and follow-up periods in the sleep laboratory (São Paulo, Brazil) using Embla N7000 recorders (Embla Systems, Broomfield, CO, USA). Participants of the HypnoLaus study were equipped with a Titanium PSG recorder (Embla Flaga, Reykjavik, Iceland) and underwent full-night PSG at their home.

At baseline, both sleep cohort studies followed the 2007 American Academy Sleep Medicine (AASM) recommended setup specifications [15]. Physiological variables evaluated during PSG included: electroencephalogram, electro-oculogram, surface electromyogram, ECG, airflow (thermistor and nasal cannula in Episono; nasal cannula in HypnoLaus), respiratory effort by inductance plethysmography belts, arterial oxygen saturation measured by pulse oximetry $\left(\mathrm{S}_{\mathrm{pO}}\right)$, snoring and body position.

At baseline, sleep staging and arousals scoring were performed according to the 2007 AASM manual in HypnoLaus [15] and according to older manuals in Episono [16]. Respiratory events were rescored according to the recommended rules of the 2012 AASM manual [17]. At Episono's follow-up, PSGs were performed and scored according to the 2012 AASM manual [17]. OSA severity was classified according to the apnoea-hypopnoea index (AHI) as mild (AHI $\geqslant 5-<15$ events $h^{-1}$ ) or moderate-to-severe (AHI $\geqslant 15$ events $\left.\cdot \mathrm{h}^{-1}\right)$. A further dichotomisation of OSA as AHI $\geqslant 15$ versus $<15$ events $\mathrm{h}^{-1}$ was performed based on the lack of substantial evidence about the cardiometabolic consequences of mild OSA [18].

\section{Outcome}

MetS was defined according to the Joint Interim Statement [2], i.e. by the presence of at least three risk factors: high blood pressure (SBP $\geqslant 130 \mathrm{mmHg}$ or $\mathrm{DBP} \geqslant 85 \mathrm{mmHg}$, or antihypertensive medication), visceral obesity (waist circumference $\geqslant 88 \mathrm{~cm}$ in females and $\geqslant 102 \mathrm{~cm}$ in males), high triglycerides $\left(\geqslant 1.7 \mathrm{mmol} \cdot \mathrm{L}^{-1}\right.$ or fibrates or nicotinic acid medication), low HDL levels $\left(<1.30 \mathrm{mmol} \cdot \mathrm{L}^{-1}\right.$ in females and $<1.03 \mathrm{mmol} \cdot \mathrm{L}^{-1}$ in males or fibrates or nicotinic acid medication) and high fasting plasma glucose $\left(\geqslant 5.6 \mathrm{mmol} \cdot \mathrm{L}^{-1}\right.$ or antidiabetic medication). In the Episono cohort, due to the high ethnical miscegenation, we considered additional cutoffs for waist circumference in those who declared themselves as Asian: $\geqslant 80 \mathrm{~cm}$ in females or $\geqslant 90 \mathrm{~cm}$ in males, as recommended by World Health Organization [19]. The difference between the number of MetS components between follow-up and baseline was denominated as changes in MetS components.

\section{Comorbidities and insulin resistance}

Hypertension was defined as SBP $\geqslant 140 \mathrm{mmHg}$ and/or DBP $\geqslant 90 \mathrm{mmHg}$ and/or use of antihypertensive medication. Diabetes was defined by fasting plasma glucose levels $\geqslant 7.0 \mathrm{mmol} \cdot \mathrm{L}^{-1}$ and/or presence of antidiabetic medication [20]. Insulin resistance was evaluated using the homeostatic model assessment of insulin resistance (HOMA-IR), defined as fasting serum insulin $\left(\mu \mathrm{U} \cdot \mathrm{mL}^{-1}\right) \times$ fasting plasma glucose $\left(\mathrm{mmol} \cdot \mathrm{L}^{-1}\right) / 22.5[21]$.

\section{Inclusion criteria}

Inclusion criteria were: 1) absence of MetS at baseline, 2) complete clinical and biological data for MetS diagnosis at baseline and follow-up, and 3) total sleep time (TST) $>4 \mathrm{~h}$ (to avoid the risk of a misrepresentation of the sleep pattern) and absence of central sleep apnoea (CSA; defined as AHI $\geqslant 5$ events. $\mathrm{h}^{-1}$ and $>50 \%$ of the events as central).

\section{Statistical analysis}

Descriptive data are presented as number of participants (with percentage or 95\% confidence interval) for categorical variables, or as mean with standard deviation or median with interquartile range for continuous variables. Bivariate analyses were performed using Chi-squared and Mann-Whitney tests. Each 
sleep-related variable was tested separately as risk factors of incident MetS through multivariate logistic regression adjusted for cohort, age, BMI and number of MetS components at baseline (Model 1). Then, a fully adjusted model including age, BMI, sex with menopausal status, cohort, number of MetS components, education level, physical activity and marital status at baseline was performed (Model 2). Results from logistic regression are presented as odds ratios and $95 \%$ confidence intervals. Since OSA prevalence is different in males and females, a stratified analysis was performed. To understand the mechanisms underlying the effect of OSA on incident MetS, multivariate-adjusted logistic regression analyses were performed with the incidence of each MetS component as an independent outcome.

Mediation analysis adjusting for confounders was performed to calculate the direct and indirect effects of OSA on the changes of the number of MetS components by testing possible mediators based on their biological plausibility. The direct effect ( $c^{\prime}$ pathway) indicates the effect of OSA on the changes of the number of MetS components taking into consideration the mediator in the model. Mediation effect was assessed by bootstrapping with 1000 replications and the Sobel test. Results of the tested mediators are presented as indirect effect coefficient $(b)$ with standard error.

From 1046 participants with OSA (AHI $>5$ events $\cdot h^{-1}$ ), only $4.2 \% \quad(n=44)$ self-reported being on continuous positive airway pressure (CPAP) treatment. However, as sensitivity analysis did not show any impact on the results when including or excluding those subjects, we included all individuals in the results. All analyses were performed using SPSS version 21 (IBM, Armonk, NY, USA) with a statistical significance set as a two-sided test with $\mathrm{p}<0.05$. For mediation analysis, we used the PROCESS procedure for SPSS version 2.16 .3 (model 4).

\section{Results}

\section{Studied population}

Of the initial 3236 participants of both cohorts, 534 (16.5\%) were dropped from the follow-up study due to death or consent withdrawal. There were no significant differences between included and excluded participants regarding BMI, MetS and sex distribution. Conversely, excluded participants were younger, single, with lower AHI and education level, and mostly from the Episono cohort (supplementary table S1).

A comparison of demographic characteristics showed some differences between the cohorts. HypnoLaus had a higher prevalence of MetS at the baseline evaluation compared with Episono (30.7\% versus 25.6\%). The mean \pm SD age $(54.3 \pm 16.6$ versus $39.5 \pm 19.5$ years $)$ as well as the prevalence of AHI $\geqslant 15$ events. $h^{-1}$ (27.2\% versus $13.2 \%$ ) were higher in HypnoLaus. However, the incidence of MetS was greater in Episono (5.6\% per year) compared with HypnoLaus ( $1.5 \%$ per year) and associated with older age. In addition, the incidence of MetS was associated with male sex and low education level only in HypnoLaus (supplementary table S2).

After exclusion of participants with missing data $(n=20)$, baseline MetS $(n=768)$, CSA $(n=4)$ and TST $<4 \mathrm{~h}$ $(\mathrm{n}=57), 1853$ participants were included in our study (figure 1). The mean \pm SD age and BMI in the merged sample were $51.9 \pm 13.1$ years and $24.9 \pm 3.7 \mathrm{~kg} \cdot \mathrm{m}^{-2}$, respectively. Approximately $56 \%$ were female and $88 \%$ were White/Caucasian ethnicity.

After a mean \pm SD follow-up of $5.9 \pm 1.3$ years, the incidence of MetS was $17.2 \%(n=318)$. Participants who developed MetS were younger, and presented a higher BMI, a lower frequency of post-menopausal females and White/Caucasian ethnicity, and a higher frequency of middle education level, Asian, Mixed and Black ethnicities, and married individuals compared with those who did not (table 1).

At baseline, participants who developed MetS had a higher number of MetS components as well as higher proportions of diabetes, hypertension, dyslipidaemia, visceral obesity, antihypertensive and antidiabetic medication use, waist circumference, SBP, DBP, insulin, HOMA-IR, and triglycerides levels compared with those who did not (table 1). Additionally, we observed a lower frequency of weekly and daily alcohol consumption as well as lower levels of total, LDL and HDL cholesterol in participants who developed MetS compared with those who did not.

Table 2 shows the univariate analysis of the sleep parameters according to the incidence of MetS. At baseline, participants who developed MetS presented lower values of TST, sleep efficiency, rapid eye movement sleep and arousal index, and higher values of time with $\mathrm{SpO}_{2}<90 \%$ and ESS score compared with those who did not. Participants who developed MetS also had a higher proportion of moderate-to-severe OSA and a lower proportion of mild OSA compared with those who did not.

\section{Sleep and incident MetS}

Table 3 shows the effect of sleep parameters on incident MetS after adjustment for cohort, age, BMI and number of MetS components at baseline (Model 1). Participants with moderate-to-severe OSA had a 


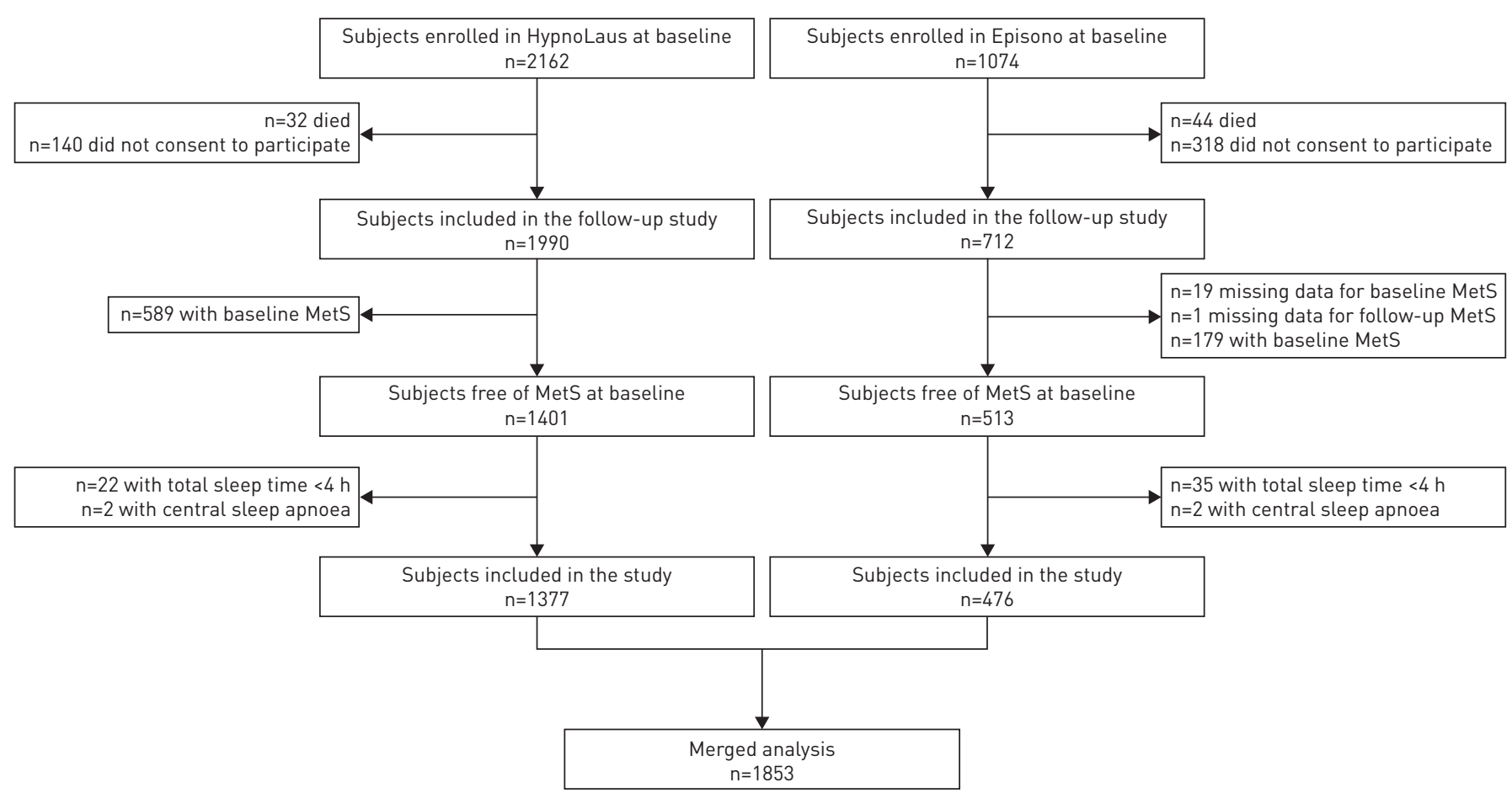

FIGURE 1 Flowchart of study design showing the selection process from initial participant enrolment to final subset analysis. MetS: metabolic syndrome.

2-fold increased risk of developing MetS compared with no OSA. After inclusion of age, BMI, sex with menopausal status, cohort, number of MetS components, education level, marital status, physical activity and ethnicity, the effect of moderate-to-severe OSA remained significant (Model 2). Similar findings were obtained when the analysis was split in male and female subsamples (supplementary table S3).

\section{Components of MetS affected by OSA status}

Among the five components of MetS (figure 2), we found a significant effect of moderate-to-severe OSA only on the incidence of visceral obesity (OR 1.65, 95\% CI 1.01-2.71) when compared with no OSA.

\section{Mediators between OSA and MetS}

Mediation analyses using OSA as the independent variable and change in MetS components as the outcome were performed adjusting for cohort, BMI, age, sex and number of MetS components at baseline (table 4). Among sleep parameters, mediation was significant only for time with $\mathrm{SpO}_{2}<90 \%$. OSA was a significant predictor of time with $\mathrm{SpO}_{2}<90 \%$ ( $b \pm$ SE 3.334 $\left.\pm 0.489 ; \mathrm{p}<0.0001\right)$, which in turn was a significant predictor of the changes in the number of MetS components ( $b \pm \mathrm{SE} 0.005 \pm 0.002 ; \mathrm{p}=0.038$ ), supporting the mediational hypothesis. OSA remained a significant predictor of the increase in the number of MetS components after controlling for time with $\mathrm{SpO}_{2}<90 \%$ ( $\left.b \pm \mathrm{SE} 0.183 \pm 0.046 ; \mathrm{p}<0.0001\right)$, indicating partial mediation (figure 3). Among cardiometabolic parameters, mediation was significant for the increase from baseline to follow-up of waist circumference, fasting glucose and insulin levels, and HOMA-IR. OSA was a significant predictor of increased waist circumference, fasting glucose, fasting insulin and HOMA-IR, which in turn were significant predictors of changes in the number of MetS components. OSA did not remain a significant predictor of changes in the number of MetS components after controlling for each of these mediators, indicating total mediation (figure 3). In addition, we verified that OSA increased waist circumference and HOMA-IR (but not glucose levels) through partial mediation of time with $\mathrm{SpO}_{2}<90 \%$ (figure 4).

\section{Evolution of sleep parameters and MetS incidence}

Noting that the Episono participants also underwent full PSG at follow-up, we sought to confirm our findings using the evolution of sleep parameters between baseline and follow-up as possible risk factors of MetS incidence (supplementary table S4). After adjustment for sex, age, baseline BMI, number of MetS components and difference in BMI, we found significant effects of the difference in time with $\mathrm{SpO}_{2}<90 \%$ and in the oxygen desaturation index (ODI) on the risk of developing MetS. Each $10 \%$ increase in time 
TABLE 1 Baseline sample characteristics according to the incidence of metabolic syndrome (MetS)

\begin{tabular}{|c|c|c|c|c|}
\hline & \multicolumn{2}{|c|}{ Incident MetS } & \multirow[t]{2}{*}{$\mathrm{n}$} & \multirow[t]{2}{*}{ p-value } \\
\hline & No & Yes & & \\
\hline Subjects & 1535 & 318 & & \\
\hline Age years & $52.2 \pm 17.6$ & $46.9 \pm 17.7$ & 1853 & $<0.0001$ \\
\hline BMI $\mathbf{k g} \cdot \mathrm{m}^{-2}$ & $24.2 \pm 4.4$ & $27.0 \pm 4.7$ & 1843 & $<0.0001$ \\
\hline$<25$ (normal) & $60.4(921)$ & $25.1(80)$ & & $<0.0001$ \\
\hline 25-29 (overweight) & $34.4(524)$ & $55.7(177)^{\#}$ & & \\
\hline$\geqslant 30$ (obese) & $5.2(80)$ & $19.2(61)^{\#}$ & & \\
\hline Sex & & & 1853 & 0.127 \\
\hline Male & $43.1(662)$ & $47.8(152)$ & & \\
\hline Female & 56.9 (873) & $52.2(166)$ & & \\
\hline Ethnicity & & & 1853 & $<0.0001$ \\
\hline White/Caucasian & 91.9 (1412) & $69.5(221)^{\#}$ & & \\
\hline Mixed & $3.5(53)$ & $17.6(56)^{\#}$ & & \\
\hline Black & $2.8(43)$ & $9.4(30)^{\#}$ & & \\
\hline Indian & $0.4(6)$ & $0.6(2)$ & & \\
\hline Asian & $0.7(11)$ & $2.2(7)^{\#}$ & & \\
\hline Other & $0.7(10)$ & $0.6(2)$ & & \\
\hline Menopausal status & & & 1010 & $<0.0001$ \\
\hline Pre-menopausal & 22.3 (337) & $29.9(94)$ & & \\
\hline Post-menopausal & $33.8(511)$ & $21.7(68)$ & & \\
\hline Education level & & & 1848 & 0.006 \\
\hline Low & $43.2(661)$ & $44.2(140)$ & & \\
\hline Middle & $31.7(486)$ & $38.5(122)^{\#}$ & & \\
\hline High & $25.1(384)$ & $17.3(55)^{\#}$ & & \\
\hline Marital status & & & 1842 & 0.009 \\
\hline Single & 21.3 (325) & $22.4(70)$ & & \\
\hline Married & $59.0(902)$ & $65.8(206)^{\#}$ & & \\
\hline Divorced/separated & $16.4(252)$ & $10.5(33)^{\#}$ & & \\
\hline Widowed & $3.3(50)$ & $1.3(4)$ & & \\
\hline MetS components $\mathrm{n}$ & $1.0 \pm 2.0$ & $2.0 \pm 1.0$ & 1838 & $<0.0001$ \\
\hline \multicolumn{5}{|l|}{ Comorbidities } \\
\hline Diabetes & 2.5 (39) & $7.6(24)$ & 1851 & $<0.0001$ \\
\hline Hypertension & $25.3(386)$ & $38.4(121)$ & 1843 & $<0.0001$ \\
\hline \multicolumn{5}{|l|}{ Medications } \\
\hline Antihypertensive & $12.7(195)$ & $20.1(64)$ & 1853 & 0.001 \\
\hline Antidiabetic & $1.0(16)$ & $3.5(11)$ & 1853 & 0.001 \\
\hline Hypolipidaemic & $11.7(180)$ & $11.0(35)$ & 1853 & 0.715 \\
\hline Smoking & & & 1798 & 0.346 \\
\hline No & $81.1(1214)$ & 78.8 (238) & & \\
\hline Yes & $18.9(282)$ & $21.2(64)$ & & \\
\hline Alcohol consumption & & & 1560 & $<0.0001$ \\
\hline Never or rarely & $28.1(359)$ & $52.6(149)^{\#}$ & & \\
\hline Weekly & $51.1(653)$ & $36.4(103)^{\#}$ & & \\
\hline Daily & $20.8(265)$ & $11.0(31)^{\#}$ & & \\
\hline Physical activity & & & 1839 & $<0.0001$ \\
\hline No & 74.5 (488) & $87.3(1034)$ & & \\
\hline Yes & $25.5(167)$ & $12.7(150)$ & & \\
\hline Waist circumference $\mathrm{cm}$ & $85.1 \pm 14.9$ & $90.3 \pm 15.8$ & 1851 & $<0.0001$ \\
\hline SBP mmHg & $119.5 \pm 20.0$ & $120.0 \pm 20.3$ & 1851 & $<0.0001$ \\
\hline DBP mmHg & $75.5 \pm 12.5$ & $80.0 \pm 12.8$ & 1851 & $<0.0001$ \\
\hline Fasting glucose $\mathrm{mmol} \cdot \mathrm{L}^{-1}$ & $5.4 \pm 0.6$ & $5.4 \pm 0.7$ & 1851 & 0.577 \\
\hline Fasting insulin pmol. $\mathrm{L}^{-1}$ & $38.9 . \pm 27.8$ & $60.1 \pm 47.2$ & 1838 & $<0.0001$ \\
\hline HOMA-IR & $1.3 \pm 1.0$ & $2.1 \pm 1.8$ & 1838 & $<0.0001$ \\
\hline 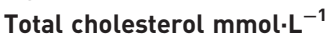 & $5.5 \pm 1.4$ & $5.1 \pm 1.5$ & 1851 & $<0.0001$ \\
\hline HDL cholesterol $\mathrm{mmol} \cdot \mathrm{L}^{-1}$ & $1.7 \pm 0.6$ & $1.4 \pm 0.4$ & 1851 & $<0.0001$ \\
\hline LDL cholesterol $\mathrm{mmol} \cdot \mathrm{L}^{-1}$ & $3.3 \pm 1.2$ & $3.1 \pm 1.3$ & 1849 & 0.008 \\
\hline Triglycerides mmol. $\mathrm{L}^{-1}$ & $1.0 \pm 0.6$ & $1.2 \pm 0.6$ & 1851 & $<0.0001$ \\
\hline hs-CRP nmol. $\mathrm{L}^{-1}$ & $76.2 \pm 128.6$ & $29.5 \pm 88.6$ & 1809 & $<0.0001$ \\
\hline
\end{tabular}

Data are presented as $n$, mediantinterquartile interval or $\%(n)$ within each group, unless otherwise stated. BMI: body mass index; SBP: systolic blood pressure; DBP: diastolic blood pressure; HOMA-IR: homeostatic model assessment of insulin resistance; HDL: high-density lipoprotein; LDL: low-density lipoprotein; hs-CRP: high-sensitivity $\mathrm{C}$-reactive protein. ${ }^{\#}$ : adjusted residual $>|2|$. Data were analysed with bivariate tests (Chi-squared or Mann-Whitney test). $p<0.05$ considered statistically significant. 
TABLE 2 Baseline sleep parameters according to incidence of metabolic syndrome (MetS)

\begin{tabular}{|c|c|c|c|c|}
\hline & \multicolumn{2}{|c|}{ Incident MetS } & \multirow[t]{2}{*}{$\mathbf{n}$} & \multirow[t]{2}{*}{ p-value } \\
\hline & No & Yes & & \\
\hline Subjects & 1535 & 318 & & \\
\hline TST min & $402.0 \pm 87.5$ & $366.5 \pm 91.9$ & 1853 & $<0.0001$ \\
\hline Sleep efficiency \% & $88.7 \pm 11.4$ & $86.9 \pm 12.1$ & 1853 & 0.002 \\
\hline N3 sleep \% & $20.3 \pm 10.4$ & $20.5 \pm 9.5$ & 1853 & 0.469 \\
\hline REM sleep \% & $22.3 \pm 7.3$ & $20.3 \pm 8.2$ & 1853 & $<0.0001$ \\
\hline Arousal index events $\cdot \mathrm{h}^{-1}$ & $16.5 \pm 11.1$ & $14.6 \pm 13.9$ & 1853 & $<0.0001$ \\
\hline AHI events· $h^{-1}$ & $6.1 \pm 11.2$ & $6.6 \pm 14.0$ & 1853 & 0.065 \\
\hline$<5$ & 43.5 (668) & 43.7 (139) & & 0.004 \\
\hline$\geqslant 5-<15$ & $34.2(525)$ & $26.4(84)^{\#}$ & & \\
\hline$\geqslant 15$ & $22.3(342)$ & $29.9(95)^{\#}$ & & \\
\hline Mean $\mathrm{SpO}_{2} \%$ & $94.9 \pm 2.1$ & $94.9 \pm 2.4$ & 1853 & 0.893 \\
\hline Lowest $\mathrm{SpO}_{2} \%$ & $89.0 \pm 7.0$ & $88.0 \pm 7.0$ & 1853 & 0.811 \\
\hline $3 \%$ ODI events $\cdot \mathrm{h}^{-1}$ & $5.5 \pm 10.8$ & $4.9 \pm 12.9$ & 1850 & 0.142 \\
\hline Time with $\mathrm{SpO}_{2}<90 \% \%$ & $0.0 \pm 0.4$ & $0.0 \pm 0.8$ & 1832 & 0.007 \\
\hline Self-reported sleep duration $h$ & $7.0 \pm 2.0$ & $7.0 \pm 2.0$ & 1793 & 0.115 \\
\hline ESS score & $6.0 \pm 5.0$ & $8.0 \pm 7.0$ & 1755 & $<0.0001$ \\
\hline
\end{tabular}

Data are presented as $\mathrm{n}$, median \pm interquartile interval or $\%(\mathrm{n})$ within each group, unless otherwise stated. TST: total sleep time; N3: slow wave; REM: rapid eye movement; $\mathrm{AHI}$ : apnoea-hypopnoea index; $\mathrm{SpO}_{2}$ : arterial oxygen saturation measured by pulse oximetry; ODI: oxygen desaturation index; ESS: Epworth Sleepiness Scale. \#: adjusted residual $>|2|$. Data were analysed with bivariate tests (Chi-squared or Mann-Whitney test). $p<0.05$ considered statistically significant.

with $\mathrm{SpO}_{2}<90 \%$ and each 10 events.h ${ }^{-1}$ increase of ODI were associated with $36 \%$ and $22 \%$ higher risk of developing MetS, respectively.

\section{Bidirectional relationship between OSA and MetS}

To assess whether the relationship between OSA and MetS could be bidirectional, a subset analysis was performed in 547 participants free of OSA from the Episono cohort (supplementary table S5). After

TABLE 3 Sleep predictors of incident metabolic syndrome (MetS)

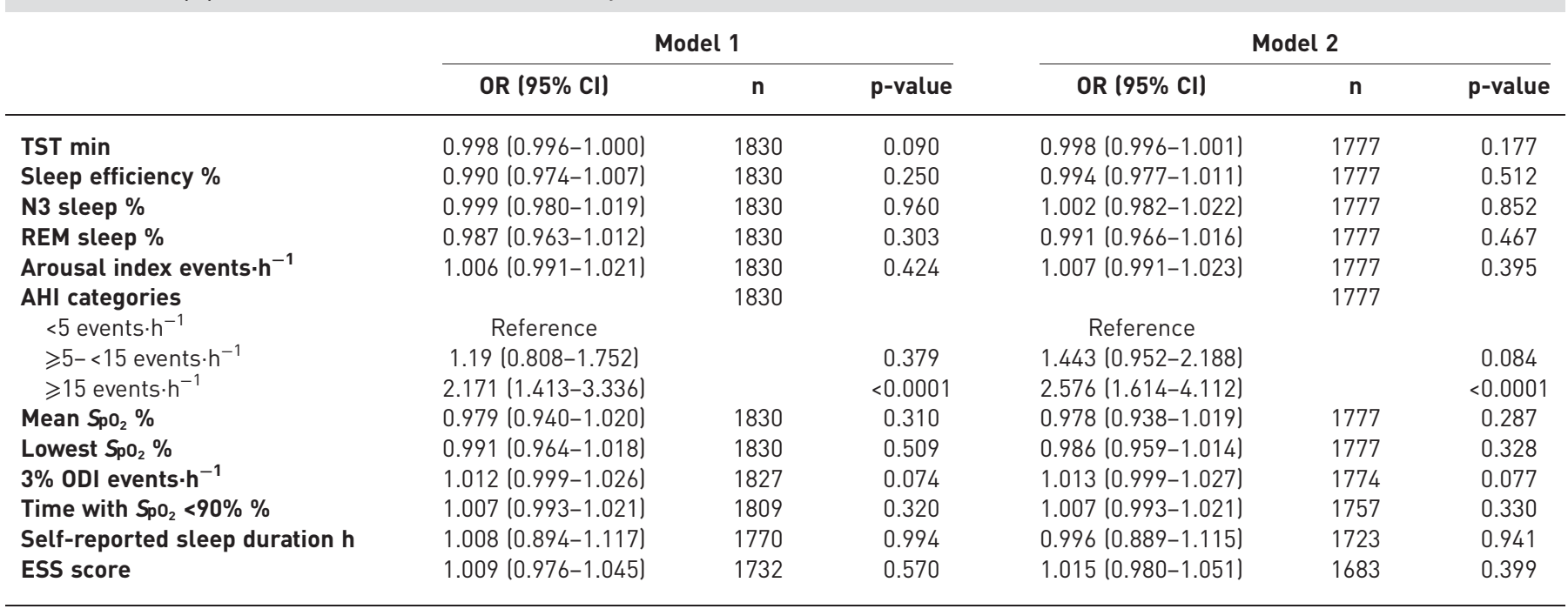

TST: total sleep time; N3: slow wave; REM: rapid eye movement; $\mathrm{AHI}$ : apnoea-hypopnoea index; $\mathrm{SpO}_{2}$ : arterial oxygen saturation measured by pulse oximetry; ODI: oxygen desaturation index; ESS: Epworth Sleepiness Scale. Data were analysed using multivariate logistic regression. Adjustment for cohort, age, body mass index and number of MetS components at baseline (Model 1); and age, sex with menopausal status, body mass index, cohort, ethnicity, education level, marital status, physical activity and number of MetS components (Model 2). p<0.05 considered statistically significant. 


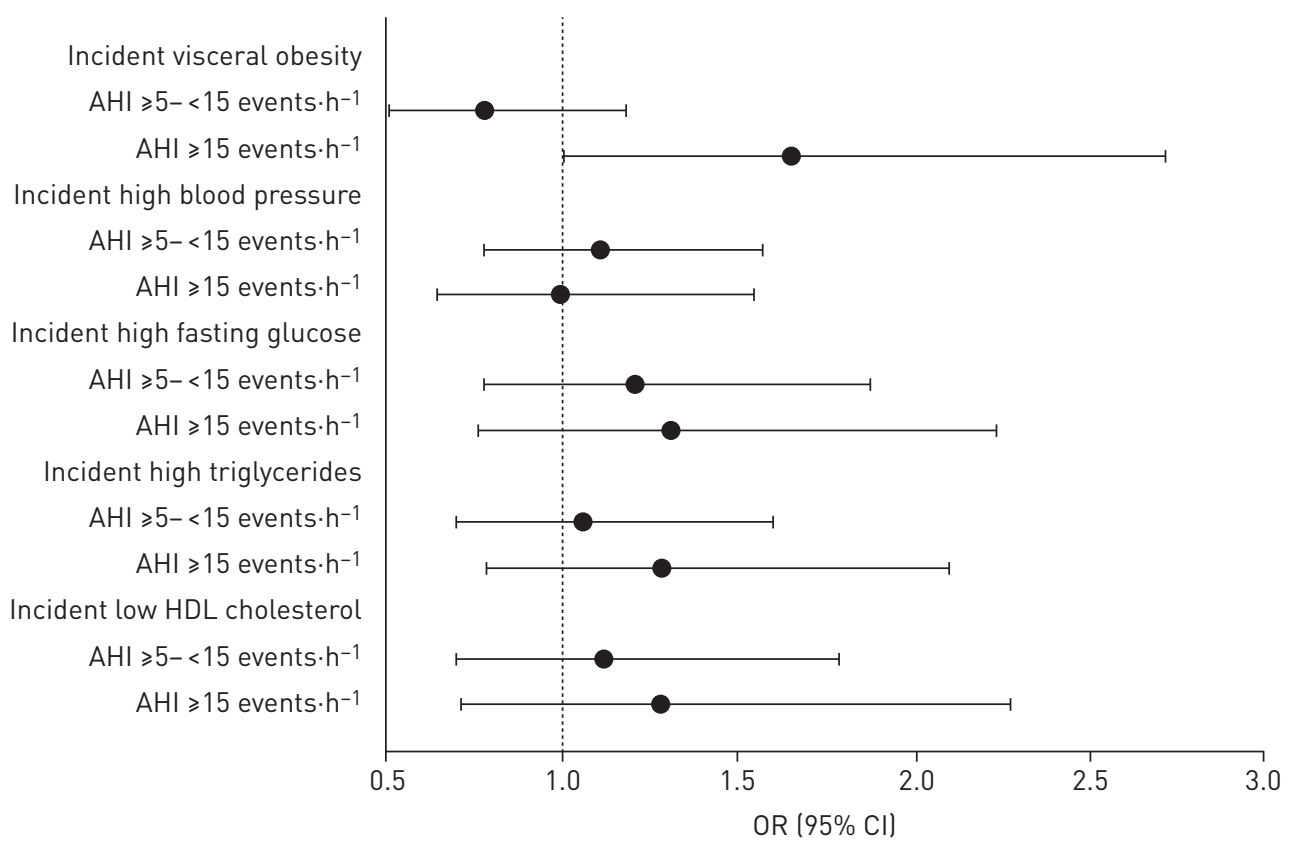

FIGURE 2 Effect of obstructive sleep apnoea severity by apnoea-hypopnoea index (AHI) categories on the risk of developing components of metabolic syndrome (MetS). HDL: high-density lipoprotein. Results were analysed using multivariate logistic regression with adjustment for age, sex, body mass index, cohort and number of MetS components. For each MetS component, analyses were restricted to participants devoid of the condition at baseline. Number of participants included: incident visceral obesity $n=1485$, incident high blood pressure $n=1175$, incident high fasting plasma glucose $n=1173$, incident high triglycerides $n=1625$ and incident low HDL cholesterol $n=1698$.

adjustment for sex, age and baseline AHI, the presence of baseline MetS was significantly associated with incident OSA (OR 1.92, 95\% CI 1.13-3.25). Nevertheless, with additional adjustment for baseline BMI, MetS was no longer a significant predictor of incident OSA.

\section{Discussion}

To the best of our knowledge, this is the first prospective study to evaluate the impact of OSA and other objectively assessed sleep parameters on the incidence of MetS. Our results show a 6-year MetS incidence of $17.2 \%$ in this large multiethnic population-based sample. Moderate-to-severe OSA was independently associated with 2-fold increased risk of developing MetS, mainly through an increase in waist circumference mediated by nocturnal hypoxaemia. On the contrary, baseline MetS was not an independent predictor of incident OSA since its effect was mainly attributable to baseline BMI.

Previous cross-sectional studies have demonstrated an independent association between OSA and MetS [8]. Dose-response relationships between OSA severity and some components of MetS have also been shown $[11,22]$.

In a prospective community-based study, only loud snoring among several subjective sleep symptoms was independently associated with increased risk of MetS [23]. In a subset of 290 participants of that study, AHI was measured using a portable monitor and showed a significant association with incident MetS after adjustment for baseline sociodemographic and lifestyle factors. Those results are in line with ours, although Troxel et al. [23] did not adjust their analysis for the baseline number of MetS components. Recently, a large prospective Korean population-based study assessed the combined effects of OSA (AHI $>10$ events $\cdot \mathrm{h}^{-1}$ ) and inflammation (high-sensitivity (hs)-CRP levels $>75$ th percentile) on the risk of developing MetS [24], and showed that the coexistence of both baseline OSA and high hs-CRP levels increased the risk of developing MetS over 6 years. Although KIM et al. [24] suggested a possible incremental effect of these factors, baseline OSA or high hs-CRP levels by themselves were also independently associated with a higher risk of MetS. In our results, although hs-CRP was not a significant mediator of the relationship between OSA and incident MetS (table 4), we found that its increase from baseline to follow-up was greater in the OSA group that developed MetS compared with the others (data not shown). 


\begin{tabular}{|c|c|c|c|}
\hline & $b \pm \mathrm{SE}(95 \% \mathrm{CI})$ & $\mathbf{n}$ & p-value \\
\hline \multicolumn{4}{|l|}{ Baseline sleep parameters } \\
\hline TST min & $0 \pm 0.002(-0.005-0.005)$ & 1805 & 0.971 \\
\hline Sleep efficiency $\%$ & $0.002 \pm 0.003(-0.003-0.01)$ & 1805 & 0.543 \\
\hline N3 sleep \% & $-0.005 \pm 0.006(-0.018-0.007)$ & 1805 & 0.454 \\
\hline REM sleep \% & $0.001 \pm 0.04(-0.007-0.007)$ & 1805 & 0.879 \\
\hline Arousal index events $\cdot \mathrm{h}^{-1}$ & $0.001 \pm 0.018(-0.034-0.037)$ & 1805 & 0.938 \\
\hline Mean $\mathrm{SpO}_{2} \%$ & $0.004 \pm 0.012(-0.002-0.034)$ & 1805 & 0.304 \\
\hline Lowest $\mathrm{SpO}_{2} \%$ & $0.004 \pm 0.015(-0.027-0.033)$ & 1805 & 0.762 \\
\hline $3 \%$ ODI events $\cdot \mathrm{h}^{-1}$ & $0.005 \pm 0.039(-0.067-0.089)$ & 1802 & 0.887 \\
\hline Time with $\mathrm{SpO}_{2}<90 \% \%$ & $0.018 \pm 0.006(0.008-0.033)$ & 1784 & 0.008 \\
\hline \multicolumn{4}{|c|}{ Follow-up cardiometabolic parameters } \\
\hline Waist circumference $\mathrm{cm}$ & $0.072 \pm 0.022(0.029-0.118)$ & 1805 & 0.002 \\
\hline SBP $\mathrm{mmHg}$ & $0.021 \pm 0.016(-0.014-0.053)$ & 1802 & 0.207 \\
\hline $\mathrm{DBP} \mathrm{mmHg}$ & $0.006 \pm 0.016(-0.028-0.037)$ & 1805 & 0.748 \\
\hline Total cholesterol mmol $\cdot \mathrm{L}^{-1}$ & $-0.006 \pm 0.006(-0.019-0.004)$ & 1805 & 0.31 \\
\hline $\mathrm{HDL}$ cholesterol $\mathrm{mmol} \cdot \mathrm{L}^{-1}$ & $0.009 \pm 0.013(-0.016-0.034)$ & 1805 & 0.45 \\
\hline LDL cholesterol $\mathrm{mmol} \cdot \mathrm{L}^{-1}$ & $-0.006 \pm 0.005(-0.018-0.002)$ & 1798 & 0.243 \\
\hline Triglycerides $\mathrm{mmol} \cdot \mathrm{L}^{-1}$ & $0.013 \pm 0.022(-0.023-0.066)$ & 1805 & 0.447 \\
\hline Fasting glucose $\mathrm{mmol} \cdot \mathrm{L}^{-1}$ & $0.037 \pm 0.02(0-0.076)$ & 1804 & 0.033 \\
\hline Fasting insulin $\mathrm{pmol} \cdot \mathrm{L}^{-1}$ & $0.05 \pm 0.016(0.020-0.081)$ & 1783 & 0.002 \\
\hline HOMA-IR & $0.053 \pm 0.016(0.022-0.083)$ & 1781 & 0.001 \\
\hline $\mathrm{hs}-\mathrm{CRP} \mathrm{nmol} \cdot \mathrm{L}^{-1}$ & $0.006 \pm 0.005(-0.001-0.022)$ & 1669 & 0.187 \\
\hline
\end{tabular}

TST: total sleep time; N3: slow wave; REM: rapid eye movement; $\mathrm{SpO}_{2}$ : arterial oxygen saturation measured by pulse oximetry; ODI: oxygen desaturation index; SBP: systolic blood pressure; DBP: diastolic blood pressure; HDL: high-density lipoprotein; LDL: low-density lipoprotein; HOMA-IR: homeostatic model assessment of insulin resistance; hs-CRP: high-sensitivity C-reactive protein. Data were analysed with mediation regressions using the PROCESS procedure for SPSS version 2.16.3 with adjustment for sex, age, cohort, body mass index and number of MetS components at baseline. Additional covariates for SBP and DBP (use of antihypertensive medication at baseline and follow-up); total cholesterol, HDL cholesterol, LDL cholesterol and triglycerides (use of hypolipidaemic medication at baseline and follow-up); and fasting glucose, fasting insulin and HOMA-IR (use of antidiabetic medication at baseline and follow-up). For cardiometabolic parameters, each follow-up measure was additionally adjusted by its respective baseline measure. $p<0.05$ considered statistically significant.

Our data suggest that nocturnal hypoxaemia, but not sleep fragmentation, is the main mediator of the increased MetS risk induced by OSA. Higher percentage of time with $\mathrm{SpO}_{2}<90 \%$ at baseline partially mediated the effect of OSA on the increased number of MetS components as well as on the increase of waist circumference and insulin resistance. This was confirmed by a subanalysis of the Episono cohort, in which the increase in both ODI and percentage of time with $\mathrm{SpO}_{2}<90 \%$ from baseline to follow-up PSGs were the only parameters independently associated with MetS incidence, even after controlling for BMI difference. In a cross-sectional analysis of the Sleep Heart Health Study cohort, participants with respiratory disturbance index $(\mathrm{RDI})>15$ events $^{-1} \mathrm{~h}^{-1}$ showed higher HOMA-IR compared with those with RDI $<5$ and $\geqslant 5-<15$ events. ${ }^{-1}$ [25]. Among the possible sleep-related contributors, PUNJABI et al. [25] found independent associations with nocturnal hypoxaemia parameters (mean $\mathrm{SpO}_{2}$ and time with $\mathrm{S}_{\mathrm{PO}_{2}}$ $<90 \%$ ), but not with arousal index.

In our study, OSA independently contributed to a higher MetS risk mainly through an increase in waist circumference, leading to higher risk of visceral obesity. Our findings are in agreement with a previous prospective study in which OSA was associated with $0.5 \mathrm{~kg} \cdot \mathrm{m}^{-2}$ increase in BMI over 5 -year follow-up compared with no OSA [5]. Additionally, males with ODI $\geqslant 10$ events $\cdot \mathrm{h}^{-1}$ who took part in a 1 -year physical exercise intervention programme showed smaller reductions in BMI and waist circumference compared with those with ODI $<10$ events $\cdot h^{-1}$, despite similar compliance to the programme [26].

In addition to visceral obesity, our study showed that OSA also increased the number of MetS components by disturbing glucose/insulin homeostasis. Several prospective cross-sectional studies have shown an independent association between OSA and insulin resistance in individuals without type 2 diabetes [27]. Moreover, a meta-analysis of six prospective cohort studies demonstrated that OSA was independently associated with a greater risk of diabetes [28]. 

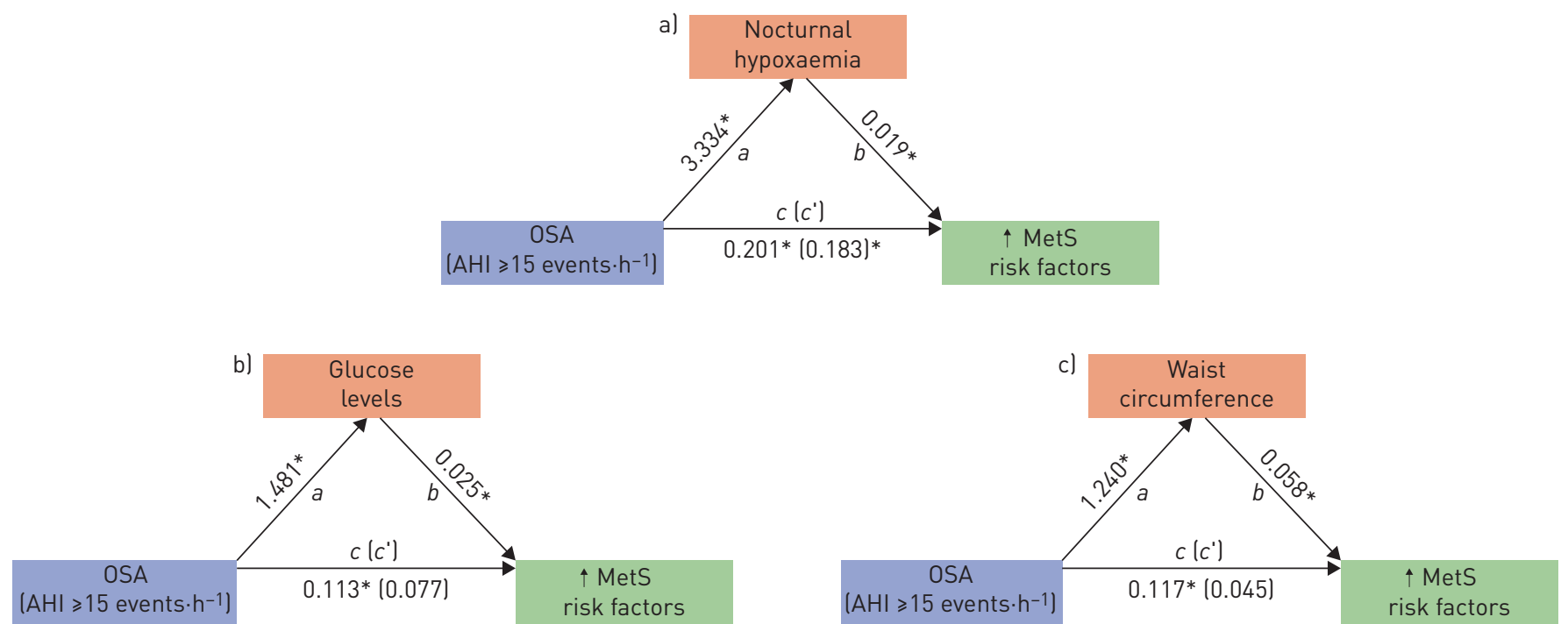

FIGURE 3 Effect of obstructive sleep apnoea (OSA) on metabolic syndrome (MetS) and its mediators. $a$ : indirect effect; $b$ : indirect effect; $c^{\prime}$ : direct effect; c: total effect; AHI: apnoea-hypopnoea index. a-c) Standardised regression coefficients for the relationship between 0SA and MetS as partially mediated by a) nocturnal hypoxaemia and totally mediated by b) glucose levels and c) waist circumference. Results adjusted for sex, age, body mass index, cohort and number of MetS components at baseline as well as for the use of antidiabetic medication at both baseline and follow-up when the mediator was the glucose level change from baseline to follow-up. *: $p<0.05$.

Although our study and others suggest a significant role of OSA in the risk of incident MetS, there is still no clear evidence supporting metabolic benefits of CPAP in OSA patients. Evidence showing modest effects of CPAP in reversing some of the MetS components [22] is somewhat contradicted by a meta-analysis of randomised controlled trials (RCTs), in which a modest increase in body weight and BMI was found after CPAP therapy [29]. Whether this inconsistency could be explained by a combination of lean mass gain and loss of visceral fat still needs to be determined. In patients with both MetS and OSA, 1-year CPAP therapy decreased the prevalence of MetS by $45 \%$ due to significant differences in waist circumference and HDL cholesterol [30]. However, there is still inconsistent evidence [31], reinforcing the need for large RCTs to determine whether treatment of OSA may help in preventing MetS. In our sample, the increased MetS risk induced by OSA does not seem to rely on blood pressure and lipid changes.
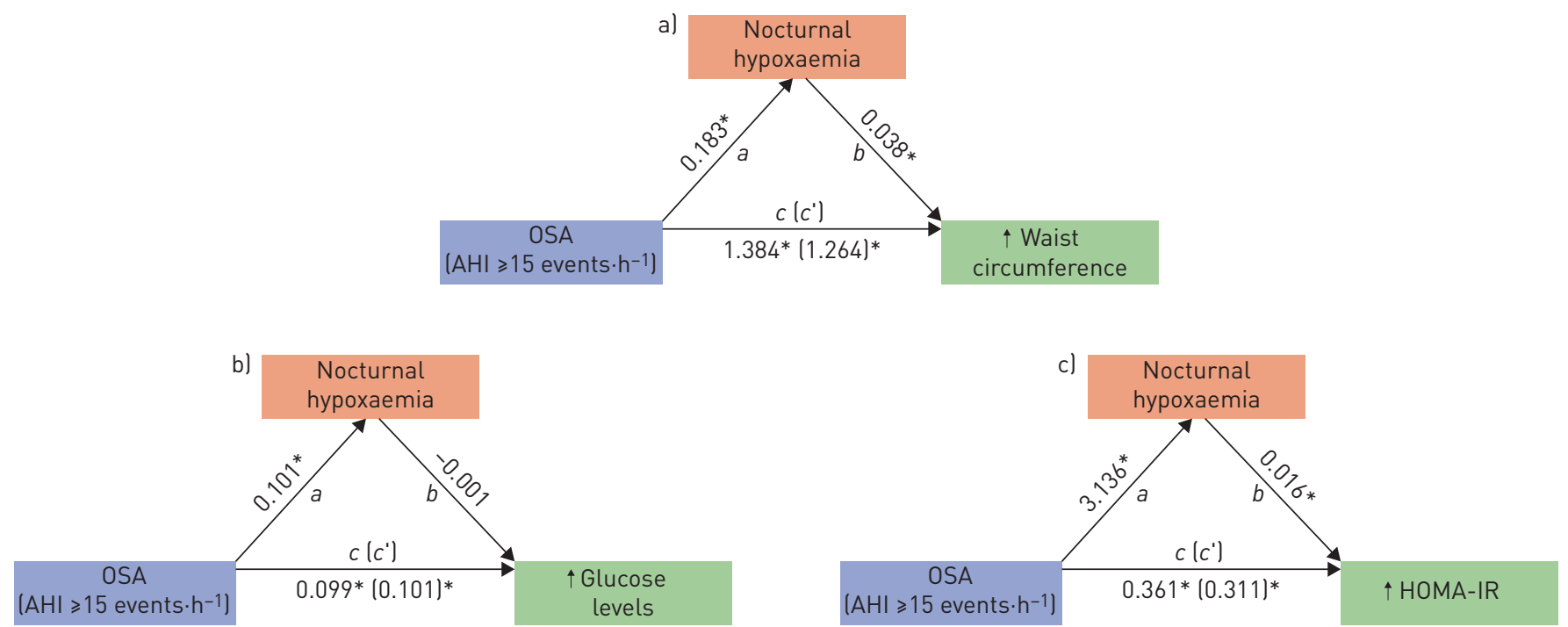

FIGURE 4 Effect of obstructive sleep apnoea (OSA) on glucose and waist circumference. $a$ : indirect effect; $b$ : indirect effect; $c$ ': direct effect; c: total effect; AHI: apnoea-hypopnoea index; HOMA-IR: homeostatic model assessment of insulin resistance. a-c) Standardised regression coefficients for the relationship between a) OSA and visceral obesity and c) OSA and insulin resistance through mediation of nocturnal hypoxaemia. OSA increased waist circumference and HOMA-IR mediated by nocturnal hypoxaemia independently from age, sex, body mass index and cohort. b) Nocturnal hypoxaemia did not mediate the effect of OSA on fasting glucose levels. *: $p<0.05$. 
Although several RCTs have demonstrated a small but significant reduction in blood pressure with CPAP therapy, it is still unclear whether it could reduce the incidence of hypertension due to a lack of studies and the presence of compliance bias [32]. The contribution of OSA to dyslipidaemia is also not well established. Meta-regressions failed to show reductions in triglycerides or increased levels of HDL cholesterol with CPAP therapy, despite decreased levels of total cholesterol having been demonstrated [33].

However, our results suggest that MetS by itself is not independently associated with an increased risk of developing OSA. The preponderant effect of BMI indicates a role for obesity on the development of OSA as expected, but it represents only one component of MetS [22]. Currently, no other prospective study has evaluated this reverse relationship between OSA and MetS.

Although both Episono and HypnoLaus cohorts were derived from unselected general populations and applied similar standardised methodologies for both sleep and metabolic assessments, there are some limitations to be acknowledged. Episono's PSGs were conducted in the sleep laboratory, whereas HypnoLaus's PSGs were performed at the participant's home. The lack of a night of adaptation in both cohorts, but mainly in Episono, could lead to changes in TST and thus affect AHI. However, in our study we elected to exclude those who had TST $<4 \mathrm{~h}$ to reduce this bias. Additionally, in the baseline evaluation of Episono, blood pressure was assessed in a single measure, which could possibly lead to an increased number of "white coat" hypertension readings.

In conclusion, our study shows that, in a multiethnic sample of two population-based samples, moderate-to-severe OSA is an independent risk factor for incident MetS, mainly through the mediation of nocturnal hypoxaemia. Given the high prevalence of OSA, and its association with high morbidity and mortality rates, our findings could have far-reaching implications for public health, but RCTs are needed to determine whether recognition and treatment of OSA may lead to a decrease in the incidence of MetS.

Acknowledgements: We thank Mehdi Tafti (Dept of Physiology, University of Lausanne, Lausanne, Switzerland) and Roberta Siuffi (Associação Fundo de Incentivo à Pesquisa, São Paulo, Brazil) for their important contributions to the HypnoLaus and Episono studies, respectively.

Author contributions: C. Hirotsu, J. Haba-Rubio, S.M. Togeiro, L.F. Drager, L. Bittencourt, R. Heinzer and S. Tufik designed the study. C. Hirotsu, J. Haba-Rubio, L. Bittencourt, R. Heinzer and S. Tufik were the study investigators, enrolled patients and collected the study data. All authors contributed equally to interpretation of the data, participated in the critical review and revision of the manuscript, and granted final approval for submission.

Conflict of interest: C. Hirotsu has nothing to disclose. J. Haba-Rubio has nothing to disclose. S.M. Togeiro has nothing to disclose. P. Marques-Vidal has nothing to disclose. L.F. Drager has nothing to disclose. P. Vollenweider reports grants from GlaxoSmithKline, during the conduct of the study. G. Waeber has nothing to disclose. L. Bittencourt has nothing to disclose. S. Tufik has nothing to disclose. R. Heinzer reports receiving fees as a member of the medical advisory boards of Nightbalance and Rhythm as well as grants from Fondation Leenaards and the Ligue Pulmonaire Vaudoise.

Support statement: The CoLaus study was and is supported by research grants from GlaxoSmithKline, the Faculty of Biology and Medicine of Lausanne (FBM), and the Swiss National Science Foundation (SNF grants 33CSCO-122661, 33CS30-139468 and 33CS30-148401). The Episono study was supported by research grants from Associação Fundo de Incentivo à Pesquisa (AFIP) and São Paulo Research Foundation (FAPESP grant 2014/15259-2). Funding information for this article has been deposited with the Crossref Funder Registry.

\section{References}

Eckel RH, Grundy SM, Zimmet PZ. The metabolic syndrome. Lancet 2005; 365: 1415-1428.

2 Alberti KG, Eckel RH, Grundy SM, et al. Harmonizing the metabolic syndrome: a joint interim statement of the International Diabetes Federation Task Force on Epidemiology and Prevention; National Heart, Lung, and Blood Institute; American Heart Association; World Heart Federation; International Atherosclerosis Society; and International Association for the Study of Obesity. Circulation 2009; 120: 1640-1645.

3 Vgontzas AN, Bixler EO, Chrousos GP. Sleep apnea is a manifestation of the metabolic syndrome. Sleep Med Rev 2005; 9: 211-224.

4 Drager LF, Lopes HF, Maki-Nunes C, et al. The impact of obstructive sleep apnea on metabolic and inflammatory markers in consecutive patients with metabolic syndrome. PLoS One 2010; 5: e12065.

5 Brown MA, Goodwin JL, Silva GE, et al. The impact of sleep-disordered breathing on body mass index (BMI): the Sleep Heart Health Study (SHHS). Southwest J Pulm Crit Care 2011; 3: 159-168.

6 Sharma SK, Kumpawat S, Goel A, et al. Obesity, and not obstructive sleep apnea, is responsible for metabolic abnormalities in a cohort with sleep-disordered breathing. Sleep Med 2007; 8: 12-17.

7 Vgontzas AN, Gaines J, Ryan S, et al. CrossTalk proposal: metabolic syndrome causes sleep apnoea. J Physiol 2016; 594: 4687-4690.

8 Qian Y, Xu H, Wang Y, et al. Obstructive sleep apnea predicts risk of metabolic syndrome independently of obesity: a meta-analysis. Arch Med Sci 2016; 12: 1077-1087.

9 Drager LF, Polotsky VY, O'Donnell CP, et al. Translational approaches to understanding metabolic dysfunction and cardiovascular consequences of obstructive sleep apnea. Am J Physiol Heart Circ Physiol 2015; 309: H1101-H1111.

10 Santos-Silva R, Tufik S, Conway SG, et al. Sao Paulo Epidemiologic Sleep Study: rationale, design, sampling, and procedures. Sleep Med 2009; 10: 679-685. 
11 Heinzer R, Vat S, Marques-Vidal P, et al. Prevalence of sleep-disordered breathing in the general population: the HypnoLaus study. Lancet Respir Med 2015; 3: 310-318.

12 Firmann M, Mayor V, Vidal PM, et al. The CoLaus study: a population-based study to investigate the epidemiology and genetic determinants of cardiovascular risk factors and metabolic syndrome. BMC Cardiovasc Disord 2008; 8: 6 .

13 Buysse DJ, Reynolds CF 3rd, Monk TH, et al. The Pittsburgh Sleep Quality Index: a new instrument for psychiatric practice and research. Psychiatry Res 1989; 28: 193-213.

14 Johns MW. A new method for measuring daytime sleepiness: the Epworth sleepiness scale. Sleep 1991; 14: 540-545.

15 American Academy of Sleep Medicine. The AASM Manual for the Scoring of Sleep and Associated Events: Rules, Terminology and Technical Specifications. 1st Edn. Westchester, AASM, 2007.

16 Sleep Disorders Atlas Task Force of the American Sleep Disorders Association. EEG arousals: scoring rules and examples: a preliminary report from the Sleep Disorders Atlas Task Force of the American Sleep Disorders Association. Sleep 1992; 15: 173-184.

17 American Academy of Sleep Medicine. The AASM Manual for the Scoring of Sleep and Associated Events: Rules, Terminology and Technical Specifications. Version 2.0. Westchester, AASM, 2012.

18 Chowdhuri S, Quan SF, Almeida F, et al. An Official American Thoracic Society Research Statement: Impact of Mild Obstructive Sleep Apnea in Adults. Am J Respir Crit Care Med 2016; 193: e37-e54.

19 Hara K, Matsushita Y, Horikoshi M, et al. A proposal for the cutoff point of waist circumference for the diagnosis of metabolic syndrome in the Japanese population. Diabetes Care 2006; 29: 1123-1124.

20 American Diabetes Association. Diagnosis and classification of diabetes mellitus. Diabetes Care 2010; 33: Suppl. 1, S62-S69.

21 Matthews DR, Hosker JP, Rudenski AS, et al. Homeostasis model assessment: insulin resistance and beta-cell function from fasting plasma glucose and insulin concentrations in man. Diabetologia 1985; 28: 412-419.

22 Phillips CL, Hoyos CM, Yee BJ, et al. CrossTalk opposing view: sleep apnoea causes metabolic syndrome. J Physiol 2016; 594: 4691-4694.

23 Troxel WM, Buysse DJ, Matthews KA, et al. Sleep symptoms predict the development of the metabolic syndrome. Sleep 2010; 33: 1633-1640.

24 Kim J, Yoon DW, Lee SK, et al. Concurrent presence of inflammation and obstructive sleep apnea exacerbates the risk of metabolic syndrome: a KoGES 6-year follow-up study. Medicine 2017; 96: e4488.

25 Punjabi NM, Shahar E, Redline S, et al. Sleep-disordered breathing, glucose intolerance, and insulin resistance: the Sleep Heart Health Study. Am J Epidemiol 2004; 160: 521-530.

26 Borel AL, Leblanc X, Almeras N, et al. Sleep apnoea attenuates the effects of a lifestyle intervention programme in men with visceral obesity. Thorax 2012; 67: 735-741.

27 Reutrakul S, Mokhlesi B. Obstructive sleep apnea and diabetes: a state of the art review. Chest 2017; 152: $1070-1086$

28 Wang X, Bi Y, Zhang Q, et al. Obstructive sleep apnoea and the risk of type 2 diabetes: a meta-analysis of prospective cohort studies. Respirology 2013; 18: 140-146.

29 Drager LF, Brunoni AR, Jenner R, et al. Effects of CPAP on body weight in patients with obstructive sleep apnoea: a meta-analysis of randomised trials. Thorax 2015; 70: 258-264.

30 Oktay B, Akbal E, Firat H, et al. CPAP treatment in the coexistence of obstructive sleep apnea syndrome and metabolic syndrome, results of one year follow up. Acta Clin Belg 2009; 64: 329-334.

31 Hoyos CM, Sullivan DR, Liu PY. Effect of CPAP on the metabolic syndrome: a randomised sham-controlled study. Thorax 2013; 68: 588-589.

32 Drager LF, McEvoy RD, Barbe F, et al. Sleep apnea and cardiovascular disease: lessons from recent trials and need for team science. Circulation 2017; 136: 1840-1850.

$33 \mathrm{Xu} \mathrm{H}$, Yi $\mathrm{H}$, Guan J, et al. Effect of continuous positive airway pressure on lipid profile in patients with obstructive sleep apnea syndrome: a meta-analysis of randomized controlled trials. Atherosclerosis 2014; 234 446-453. 